\title{
Aprendizaje Basado en Proyectos en la escuela secundaria argentina: un estudio exploratorio sobre la experiencia de profesoras y profesores en servicio
}

Corica, Ana Rosa

Aprendizaje Basado en Proyectos en la escuela secundaria argentina: un estudio exploratorio sobre la experiencia de profesoras y profesores en servicio

Revista Educación, vol. 45, núm. 1, 2021

Universidad de Costa Rica, Costa Rica

Disponible en: http://www.redalyc.org/articulo.oa?id=44064134039

DOI: https://doi.org/10.15517/revedu.v45i1.43084

\section{(c) $($ ) $\Theta \Theta$}

Esta obra está bajo una Licencia Creative Commons Atribución-NoComercial-SinDerivar 3.0 Internacional. 


\title{
Aprendizaje Basado en Proyectos en la escuela secundaria argentina: un estudio exploratorio sobre la experiencia de profesoras y profesores en servicio
}

\author{
Project-Based Learning at an Argentinian High School: An Exploratory Study on In-Service Teacher Experience
}

Ana Rosa Corica

Consejo Nacional de Investigaciones Cientificas y Técnicas

(CONICET), Núcleo de Investigación en Educación en

Ciencias y Tecnologia (NIECyT), Argentina

acorica@exa.unicen.edu.ar

(iD) https://orcid.org/0000-0002-3583-6081
DOI: https://doi.org/10.15517/revedu.v45i1.43084

Redalyc: http://www.redalyc.org/articulo.oa?id=44064134039

Recepción: 30 Julio 2020

Aprobación: 08 Octubre 2020

\section{Resumen:}

En los últimos años, en Argentina se propuso un cambio de la educación secundaria fundamentado en el enfoque del Aprendizaje Basado en Proyectos. Este trabajo tiene como objetivo conocer las condiciones y restricciones que manifiestan experimentar profesoras y profesores en servicio, al gestionar una enseñanza por Aprendizaje Basado en Proyectos en escuelas de educación secundaria en Argentina. En este trabajo se propone una investigación cualitativa de tipo exploratoria. Se desarrolló una entrevista grupal en profundidad con profesoras en ejercicio que desarrollan funciones docentes en una escuela secundaria que promueve el Aprendizaje Basado en Proyectos. Las profesoras participaron de la gestión de un proyecto sobre alimentación saludable, en el que intervinieron la totalidad de las y los docentes del año escolar en el que se desarrolló. El análisis de la entrevista se realizó a partir del análisis de categorías de carácter deductivo. Los principales resultados indican que la especialización en que son formadas las y los profesores de la escuela secundaria, basada en la parcialización del saber, y la estructura actual de la escuela secundaria condiciona y restringe el estudio interdisciplinario que requiere emprender cualquier proyecto del mundo real. Es preciso idear propuestas compatibles con una enseñanza por Aprendizaje Basado en Proyectos para la formación de profesores que conduzcan a cambiar la propia mirada sobre su profesión y las personas estudiantes. Esto conduce a una formación que no se puede fundamentar en la reproducción o almacenamiento de información, sino que busca su funcionalidad y reconstrucción en diversas situaciones.

Palabras clave: Aprendizaje Basado en Proyectos, Docentes en Servicio, Escuela Secundaria, Enseñanza.

\section{Abstract:}

In recent years, Argentina has proposed changes to Secondary Education based on a Project-Based Learning approach. The objective of this study is to explore the conditions and restrictions manifested by in-service teachers who teach Project-Based Learning at high schools in Argentina. This study is qualitative and exploratory in nature and based on an in-depth group interview with in-service high school teachers who teach Project-Based Learning. These teachers helped manage a project on healthy eating which included the faculty for the participating grade. The interview was assessed based on analysis of deductive categories. According to the results, when teachers are trained in their specialty area their knowledge becomes biased. This, added to the current structure of high schools, restrict teachers from undertaking the interdisciplinary study required to execute any real-world project. It is necessary to design teaching proposals compatible with Project-Based Learning that will encourage teachers to change their perception about their profession as well as that of their students. Such training cannot be based on reproducing or storing information, rather, it must be functional and be able to reenact real-life situations.

KeYwords: Project-Based Learning, Service Teachers, High School, Teaching.

\section{INTRODUCCIÓN}

Recientemente, en Argentina se propuso un cambio de la educación secundaria fundamentado en el enfoque del Aprendizaje Basado en Proyectos (ABP) (Ministerio de Educación de la Nación, 2017). Esto implica que las profesoras y los profesores se involucren en el proceso de estudio como mediador que interviene 
y ofrece ayuda pedagógica regulada para que las personas estudiantes construyan su propio conocimiento, desarrollando habilidades tanto a nivel individual como en interacción con sus pares.

Las propuestas ministeriales fomentan un estudio interdisciplinar, con el propósito de superar la fragmentación de la enseñanza y el aprendizaje, proponiendo el diálogo, la articulación y la vinculación entre los saberes. Los problemas que se plantean en las sociedades actuales, requieren de la integración de saberes provenientes de distintas disciplinas para ser analizados y sintetizados en un saber integrado, que posibilite crear productos, plantear interrogantes y, de esta manera, construir diferentes explicaciones o propuestas de solución a esos problemas. Se propone que las personas estudiantes aborden los hechos desde una visión global, no fragmentada, que les permita dar significado a las situaciones que se les presentan y la oportunidad de desarrollar conocimiento, al participar de manera activa. Esta perspectiva demanda a las personas docentes a desarrollar un trabajo pedagógico conjunto, colaborativo y articulado, con el fin de organizar la enseñanza desde un diálogo entre las nociones, las metodologías, los procesos y procedimientos, y la propuesta de actividades interdisciplinarias (Ministerio de Educación de la Nación, 2017). Estas actividades conllevan al desafío de superar las visiones fragmentadas para asumir una posición pedagógica, que diluya las fronteras entre las disciplinas y entre la teoría y la práctica. A través de esta propuesta no se promueve la pérdida de la especificidad, ni del saber especializado, sino que se invita a problematizar la forma en que su organización permite abordar algunos temas u otros y hacerlo de manera acorde a las necesidades de la educación secundaria (Dirección de Educación Secundaria de la Provincia de Buenos Aires, 2017).

En la provincia de Buenos Aires en Argentina, las nuevas propuestas ministeriales se han comenzado a experimentar a partir del programa Escuela Promotora (Gobierno de la Provincia de Buenos Aires, 2018). Este programa tiene como propósito el acompañamiento de naturaleza integral y progresiva de los itinerarios formativos de las personas estudiantes, enfatizando la articulación entre los niveles primario y secundario, fomentando proyectos socio-comunitarios y favoreciendo la vinculación profesional de las personas docentes con la institución. Así también, se propone contribuir a mejorar la trayectoria escolar y el aprendizaje de las personas estudiantes del nivel secundario, disminuir las tasas de repitencia y abandono escolar, incrementar la graduación y aportar al clima escolar general. El programa se compone de los siguientes elementos: un proceso de articulación entre niveles, el fortalecimiento del seguimiento de estudiantes a través de una persona docente de la institución denominada Profesor Acompañante de Trayectoria (PAT), un trabajo pedagógico institucional, que consiste en el desarrollo de una propuesta de prácticas pedagógicas a partir de saberes integrados y una evaluación colegiada. En el año 2018 se inició esta experiencia pedagógica, siendo implementada en 600 escuelas de la Provincia de Buenos Aires. La implementación comenzó por el primer año de la educación secundaria y se asignan módulos institucionales al PAT y a las profesoras y los profesores de los años escolares involucrados, con el objetivo de participar en reuniones obligatorias para la planificación, desarrollo y evaluación de proyectos.

Este trabajo tiene como objetivo conocer las condiciones y restricciones que manifiestan experimentar las y los profesores en servicio, al diseñar e implementar una enseñanza por ABP en escuelas de educación secundaria, con el propósito de evaluar procesos de formación para las y los profesores en la gestión de ABP. Interesa tomar conocimiento y profundizar acerca de los saberes que producen profesores y les permiten tomar decisiones y actuar ante determinadas situaciones propias del mundo escolar, pues Terigi (2007) indica que estos saberes construidos a lo largo de la trayectoria profesional son poco considerados, no formando parte de la preparación profesional de las personas docentes. Recuperar esos saberes prácticos, experienciales, para estudiarlos, reconstruirlos y discutirlos son fundamentales para contribuir a la formación del oficio docente.

En este trabajo se describe y analiza una entrevista desarrollada con profesoras y profesores que desempeñan funciones docentes en una escuela secundaria que forma parte del programa Escuela Promotora. Las personas participaron de la gestión de un proyecto sobre alimentación saludable, en la que intervinieron docentes del año escolar en el que se desarrolló. Interesa tomar conocimiento de la gestión de ABP por la 
propia voz de las y los profesores participantes, porque, si bien hay una amplia proliferación de experiencias de la enseñanza por ABP en diversos sistemas educativos, las investigaciones acerca de su funcionalidad y experiencias reales es todavía escasa (Torrego y Méndez, 2018). Esto es atribuido, entre otras razones, a las dificultades pedagógicas y técnicas para la aplicación de una enseñanza por ABP, el arraigo a la enseñanza tradicional y la presión por cumplir con el currículo (Ayerbe y Perales, 2020). Se torna necesario profundizar en conocer el funcionamiento de un $\mathrm{ABP}$ y las posibilidades de su gestión en contextos educativos formales de nuestro país, donde se ha comenzado a implementar en los últimos años.

\section{El Aprendizaje Basado en Proyectos}

A principios del siglo XX, William Heart Kilpatrick trazó las bases del ABP. Esta propuesta adopta una "filosofía experimental de la educación" (Kilpatrick, 1967a, p. 72), es decir, el conocimiento se adquiere a través de la experiencia. Así mismo, critica la fragmentación del conocimiento en materias, asignaturas o áreas, porque aprender aisladamente significa que "el alumno no ve o siente la utilidad o pertinencia de lo que se enseña para ningún asunto que le interesa en el presente, y por tanto no se adhiere inteligentemente a la situación actual" (Kilpatrick, 1967b, p. 49). Se propone un nuevo programa escolar en el que las áreas se reúnan "desde el punto de arranque de las necesidades del alumno" (Kilpatrick, Rugg, Washburne y Bonner, 1967, p. 29). Kilpatrick (1967b) afirma que "el aprendizaje no es nunca simple" (p. 70) y requiere conexiones con el medio social. Se busca una organización trasversal del conocimiento en el que los saberes se estudien de manera integral.

El ABP se basa en la indagación, constituyendo una pedagogía que tiene raíces constructivistas, fundamentada en los trabajos de Piaget, Dewey y Vygotsky (Dewey, 1997; Ginsburg y Opper, 1987; Vygotsky, 1962). El aprendizaje por indagación involucra la formulación de preguntas, emprender investigaciones para responder estas preguntas, analizar e interpretar datos, y aportar posibles soluciones (Bell, Urhahne, Schanze y Ploetzner, 2010).

Siguiendo a Kilpatrick (1918), el desarrollo de un ABP se conforma de cuatro fases: intención, preparación, ejecución y evaluación. Sin embargo, no todos los proyectos comparten la misma finalidad; en particular, Kilpatrick (1918) propone cuatro tipos de trabajo por proyectos según la finalidad que se persiga: elaboración de un producto final, conocer un tema y disfrutar con su conocimiento o experiencia, mejorar una técnica o habilidad concreta, o resolver un problema intelectual desafiante para el protagonista (Majó y Baqueró, 2014). En referencia a cuestiones procedimentales de un APB, Vergara (2016) establece diferentes formas mediante las que se puede generar un proyecto: interés espontáneo del estudiante, suceso o acontecimiento, los días de..., encargo, acción provocada y propuesta comunitaria. En particular, la acción provocada es una de las formas más utilizadas por las personas docentes de diferentes asignaturas, quienes eligen un tema con suficiente potencia para desarrollar entre todas estas y lo desarrolla con estudiantes.

Según Thomas (2000), los proyectos deben ser el centro del currículo y no algo periférico. El ABP no debe ser realizado en una determinada unidad didáctica, sino que debe ser el eje vertebrador mediante el cual se lleve a cabo el proceso de enseñanza-aprendizaje (Larmer y Mergendoller, 2010). Es normal que un área actúe como motor de un proyecto, sin embargo, es necesario explorar vías para que todas las áreas curriculares tengan presencia, siempre y cuando dicha presencia no sea forzada; con la finalidad de no perpetuar la clásica división entre áreas de mayor y menor reconocimiento social y curricular (López, Ugalde, Rodríguez, Rico, 2015).

Lacueva (1998) destaca que "no son proyectos todas aquellas actividades en las que el problema y la metodología ya vienen dados y donde las niñas y los niños se limitan a actuar" (p. 3), porque de esta manera los y las profesoras continuarían siendo especialistas del proceso de estudio. La enseñanza por ABP implica un cambio en la cultura escolar, que exige cultivar la cultura cooperativa entre las y los profesores con la finalidad de superar el aislamiento de la escuela tradicional. Vergara (2016) destaca que el ABP implica 
una redefinición del rol de las profesoras y los profesores; este es concebido tradicionalmente como gestor del aprendizaje de estudiantes, quien se centra en orientar, dinamizar, aportar criterios, organizar saberes, etc. En un ABP las personas estudiantes anticipan, planifican y realizan, bajo la supervisión del cuerpo docente, una actividad que requiere recurrir a nociones que refieren a distintas disciplinas (Eceizabarrena, 2018). En la enseñanza por proyectos, las personas estudiantes aprenden de su propia experiencia, buscando información, manipulándola, ordenándola y presentándola convenientemente. Las personas estudiantes plantean problemas, hipótesis, las resuelven, se confunden, reflexionan, piden ayuda, se coordinan con sus compañeras y compañeros, deciden, actúan; todo esto con la finalidad de saciar la curiosidad del tema propuesto de manera consensuada. Cada estudiante asume un papel diferente: de liderazgo, conciliador, creativo, pasivo o ácrata. A la hora de cooperar puede ocurrir que todos los miembros del grupo aporten de la misma manera, que se produzcan descompensaciones o que la cooperación desaparezca. Las profesoras y los profesores deben reconducir estas situaciones para que toda persona integrante tenga voz.

Una enseñanza por ABP también implica cambios en los espacios asignados al estudio en la escuela: la estructura temporal basada en los módulos de una hora se disipa. En la enseñanza por proyectos predomina el aula unida al grupo como único espacio de aprendizaje (Vergara, 2016).

\section{Metodología}

En este trabajo se propone una investigación cualitativa de tipo exploratoria (Hernández, Fernández y Baptista, 2014). Se desarrolló una entrevista grupal en profundidad con profesoras y profesores en ejercicio que desarrollan funciones docentes en una escuela secundaria pública que participa del programa Escuela Promotora. La entrevista en profundidad permite obtener información rica y profunda en las propias palabras de las y los actores, permitiendo el acceso a información difícil de conocer sin la mediación de quien entrevista, brindando la posibilidad de conocer a través de su relato, situaciones no directamente observables (Valles, 1999).

Para la selección del grupo de profesoras y profesores que participaron de la investigación, en primer lugar, se entrevistó a personas inspectoras y directoras de instituciones que se encuentran en el programa Escuela Promotora. Esto permitió tomar conocimiento del funcionamiento del programa en las instituciones, los proyectos que desarrollan profesores y las condiciones en las que son gestionados. Las profesoras y los profesores que intervinieron en la entrevista se caracterizan por haber participado en el desarrollo de un proyecto que involucró a la totalidad de docentes del curso en el que se llevó a cabo. Este fue el aspecto esencial por el que se optó desarrollar la investigación con estas personas docentes, siendo que, en otros grupos en los que se indagó, las profesoras y los profesores desarrollan proyectos en los que interactúan otros dos o tres profesionales. Otro de los aspectos que interesó para la selección del grupo de docentes a entrevistar es la temática abordada en el proyecto y su desarrollo con las personas estudiantes de contextos vulnerables. Las profesoras y los profesores que participaron de la entrevista se caracterizan por haber trabajado en conjunto en el desarrollo de un proyecto sobre alimentación saludable durante seis meses. El proyecto demandó el trabajo cooperativo de profesores que se ocupan de los espacios curriculares: Ciencias Sociales, Ciencias Naturales, Matemática, Prácticas del Lenguaje, Construcción de la Ciudadanía, Inglés, Educación Física y Artística. La entrevista se desarrolló con la profesora de Ciencias Sociales $(\mathrm{PH})$ cuya formación es de Historia, la profesora de Ciencias Naturales (PB) cuya formación es Biología y la profesora de Matemática (PM). La entrevista tuvo lugar en el espacio que profesores se reúnen para conversar acerca de la gestión del proyecto. Si bien, a este espacio pueden asistir la totalidad de las profesoras y los profesores que se involucran en el proyecto, el pago por su trabajo solo se realiza a dodentes que tienen cuatro horas en el curso que se desarrolla el proyecto, por lo que las profesoras y los profesores de las materias Construcción de la Ciudadanía, Educación Física, Artística e Inglés quedan exceptuados de los encuentros.

La entrevista tuvo una duración de 75 minutos, en los que se procuró conocer las condiciones y restricciones que manifiestan experimentar profesores en la gestión de proyectos en la escuela secundaria. En particular, el análisis tiene como finalidad explorar las distintas dimensiones o factores que surgen cuando un 
equipo de docentes experimenta una enseñanza por ABP. Durante la entrevista, la investigadora registró las intervenciones de las profesoras, realizó preguntas definidas desde un principio y otras nuevas que emergieron durante la conversación, con el propósito de profundizar, documentar, buscar significados e interpretar las intervenciones de las participantes. Durante la entrevista, la investigadora trató de intervenir lo menos posible, evitando dar su opinión sobre la temática; el trato a todas las participantes fue igualitario, y se buscó evidencias positivas y negativas por igual, con el objetivo de procurar la credibilidad del estudio (Savin y Major, 2013).

El análisis de la entrevista se realizó a partir de categorías que engloban las preguntas que estructuraron la entrevista. El criterio adoptado para la formulación de las categorías fue deductivo (Mejía, 2011). Se partió de categorías establecidas a priori sobre las que se formularon las preguntas fundamentales con las que se estructuró la entrevista. Por las características de la entrevista, durante la conversación emergieron otras cuestiones que profundizaron aspectos de las preguntas originales y que se identificaron con las mismas categorías.

En la Tabla 1 se indican las categorías a las que corresponden las preguntas formuladas por la entrevistadora y los objetivos que se persiguieron. La situación inicial que dio lugar a la conversación fue la formación de profesoras y profesores, siendo que una enseñanza por ABP requiere un trabajo interdisciplinar y esta formación en Argentina es monodiscipliar: esto fomenta la segmentación de las disciplinas y dificulta el estudio interdisciplinario de problemas. Una vez iniciada la conversación, la entrevistadora formuló las preguntas que se indican en la Tabla 1.

TABLA1

Categorías de análisis

\begin{tabular}{|c|c|c|}
\hline Categorías & Preguntas & Objetivos \\
\hline $\begin{array}{l}\text { Los proyectos que se desarrollan en la } \\
\text { escuela secundaria }\end{array}$ & $\begin{array}{l}\text { ¿Cómo se originaron los proyectos que } \\
\text { desarrollaron en la escuela? ¿Cuál es su opinión en } \\
\text { relación al estudio por ABP en las condiciones } \\
\text { actuales de la escuela secundaria? ¿De qué manera } \\
\text { establecieron vínculos entre el trabajo por } \\
\text { proyectos y el diseño curricular vigente para la } \\
\text { escuela secundaria? }\end{array}$ & $\begin{array}{l}\text { - Identificar cómo las profesoras y los profesores formulan } \\
\text { los proyectos. - Estudiar el alcance del ABP en las prácticas } \\
\text { desarrolladas. - Establecer los vínculos que establecen las } \\
\text { profesoras y los profesores con el ABP y el diseño curricular } \\
\text { vigente. }\end{array}$ \\
\hline $\begin{array}{l}\text { El rol de la profesora y el profesor en una } \\
\text { enseñanza por ABP }\end{array}$ & $\begin{array}{l}\text { ¿Cómo se organizan las profesoras y los profesores } \\
\text { para enseñar por ABP? ¿Qué cambió en ustedes al } \\
\text { trabajar en conjunto con otras profesoras y otros } \\
\text { profesores? }\end{array}$ & $\begin{array}{l}\text { - Establecer los procedimientos seguidos por las profesoras y y } \\
\text { los profesores en el diseño y desarrollo de un ABP. - Analizar } \\
\text { la valoración que realizan las profesoras y los profesores } \\
\text { cuando experimentan una enseñanza por ABP. }\end{array}$ \\
\hline $\begin{array}{l}\text { El rol de las y los estudiantes en una } \\
\text { enseñanza por ABP }\end{array}$ & $\begin{array}{l}\text { ¿De qué manera las y los estudiantes se } \\
\text { involucraron en los proyectos desarrollados? ¿Qué } \\
\text { diferencias encuentran en la participación de las y } \\
\text { los estudiantes en un ABP y otras formas de } \\
\text { enseñanza? }\end{array}$ & - Caracterizar el rol de las y los estudiantes en un ABP. \\
\hline $\begin{array}{l}\text { La evaluación en una enseñanza por } \\
\text { proyectos }\end{array}$ & ¿Cómo se evalúa en una enseñanza por ABP? & $\begin{array}{l}\text { - Profundizar acerca del sistema de acreditación en una } \\
\text { enseñanza por ABP. }\end{array}$ \\
\hline $\begin{array}{l}\text { La formación de las profesoras y los } \\
\text { profesores y la enseñanza por ABP }\end{array}$ & $\begin{array}{l}\text { ¿Qué formación recibieron para el desarrollo de } \\
\text { una enseñanza por ABP? }\end{array}$ & $\begin{array}{l}\text { - Describir la formación que tienen las profesoras y los } \\
\text { profesores para gestionar una enseñanza por ABP. }\end{array}$ \\
\hline
\end{tabular}

Fuente: Elaboración propia

Para el análisis de la entrevista, al audio se transcribió y se segmentó en episodios. Cada episodio fue definido según la conversación hiciera referencia a las categorías indicadas. Se establecieron 20 episodios. Se destaca que, por las características de la entrevista, algunas de las categorías fueron identificadas en más de un episodio. A continuación, se describe y analiza cada una de las categorías formuladas. 


\section{ANÁLISIS Y DISCUSIÓN DE RESULTADOS}

En esta sección se analiza la entrevista desarrollada. Para esto se describe cada categoría en función de las intervenciones de las profesoras. La descripción no sigue un orden cronológico, siendo que la conversación tuvo como objetivo principal profundizar en las experiencias de las personas participantes en la gestión de proyectos, y, debido a que la entrevista fue propuesta como semi-estructurada en diferentes episodios, se abordaron las mismas temáticas según lo convocó la conversación.

\subsection{Los proyectos que se desarrollan en la escuela secundaria}

Esta categoría reúne las intervenciones de las profesoras que se refieren a la enseñanza por ABP y la escuela secundaria actual. En particular, las nuevas propuestas ministeriales se han puesto en marcha sobre una estructura escolar que conserva los mismos diseños curriculares desde la reforma educativa argentina (Ministerio de la Nación, 2006). En el diseño curricular se indican los saberes mínimos a estudiar de cada materia para cada año escolar; las que también se encuentran confinadas a ser desarrolladas en determinado espacio temporal.

En el episodio 3 de la entrevista, las profesoras $\mathrm{PB}$ y $\mathrm{PH}$ profundizaron en las características del proyecto que se encontraban desarrollado en el momento que fueron entrevistadas. Se trató de un proyecto de alimentación saludable que se desarrolló durante 6 meses, e intervinieron la totalidad de las profesoras y los profesores de primer año. El proyecto fue propuesto por $\mathrm{PB}$, por sugerencia de la dirección de la institución, siendo que el año anterior lo había experimentado con estudiantes de primer año de la escuela (cuyas edades oscilan entre 12-13 años).

El proyecto que se encontraban desarrollando las profesoras surge de una acción provocada (Vergara, 2016), originada por un grupo de profesoras y profesores que moviliza al resto de la comunidad educativa. La potencialidad de esta modalidad en el nacimiento del proyecto es la alta motivación por parte de las profesoras y los profesores (Vergara, 2016). Las personas docentes propusieron una temática que aporte conocimiento al estudiantado y que trasciendan a sus familias, siendo que el proyecto se desarrolló con estudiantes que viven en contextos vulnerables. La atención del proyecto se ubicó en que las y los estudiantes adquieran conocimientos para su desarrollo personal y familiar más allá de lo curricular. Esto se pone de manifiesto en el episodio 3:

No solo por ahí los contenidos y hagan la barra... sino que también ellos aprendan a tener una salida laboral. No por ellos, porque ellos vayan a ir a hacer las barritas y salgan a vender, pero por ahí a la familia (...) porque son chicos de lugares muy carenciados (...) con hambre, entonces al momento con hambre, a la hora de poder dar un contenido vos le hablas de cosas y a los chicos les hace ruido la panza y no pueden pensar (...) desde ese lado, orientamos un poco más el proyecto para que vean que hay otras salidas. Vimos comidas, alimentos de antes. Ella que es de historia, alimentos que se comían antes, alimentos que se comen ahora. El tiempo que hay para elaborarlos, porque antes por ahí la mamá se quedaba en la casa y elaboraba un poco más y ahora no, todo es rápido (...) ( , comunicación personal, 1 de noviembre, 2019)

"Vino bromatología, vinieron a hablar por el tema de manipulación de alimentos." (PH, comunicación personal, 1 de noviembre, 2019) "Porque no es solo hacerlo en casa, sino todo lo que hay alrededor. La higiene, tanto del lugar donde lo vas a hacer, la conservación.” (PB, comunicación personal, 1 de noviembre, 2019).

En las intervenciones de las profesoras $\mathrm{PB}$ y $\mathrm{PH}$ se pone de manifiesto que el proyecto tiene como objetivo generar un producto final, pero durante el desarrollo de este se procura que los estudiantes adquieran conocimientos disciplinares, conociendo su funcionamiento en el desarrollo de un proyecto. Esto último también se pone de manifiesto en el episodio 14: "La profe de inglés hizo las diferentes comidas y los nombres en inglés de las comidas (...)" (PB, comunicación personal, 1 de noviembre, 2019). "Desde Práctica del Lenguaje trabajaron diferentes textos, y en este caso hicieron folletos y trabajaron directamente sobre 
enfermedades como es el colesterol, la hipertensión (...)" (PH, comunicación personal, 1 de noviembre, 2019). "Después hicieron resumen, lo pusieron en un folleto. Ella le explicó [en referencia a la profesora de Práctica del Lenguaje] cómo tenían que armar un folleto. Dónde podía haber imagen, dónde no.” (PB, comunicación personal, 1 de noviembre, 2019)

En relación con la toma de decisiones acerca de cómo gestionar el proyecto, en el episodio 3 PH destacó aspectos a considerar al iniciar un proyecto:

(...) lo arrancamos cerca del segundo trimestre porque cada una de nosotras tenía que dar los contenidos para dar pie a arrancar (...) vos necesitas dar ciertos contenidos. No podés arrancar con el proyecto desde la nada. Entonces tenés que tener en cada una de las materias la base para poder arrancar ( $\mathrm{PH}$, comunicación personal, 1 de noviembre, 2019).

En la intervención de PH se advierte que una vez definida la temática a trabajar, cada profesora o profesor, para su materia, establece qué nociones necesitan saber las personas estudiantes para luego comenzar el desarrollo del proyecto. La etapa de planificación del proyecto sobre alimentación parecería ser ocupada por las y los profesores, dado que son los que deciden qué proyecto realizar y qué conocimientos estudiar previamente para el desarrollo de este. En esta propuesta no encuentra lugar la indagación por parte de estudiantes. Para Jones, Rasmussen y Moftt (1997), el ABP comprende un conjunto de tareas basado en el estudio de preguntas o problemas que demandan a las personas estudiantes la toma de decisiones e involucrarse en procesos de investigación, favoreciendo la oportunidad de trabajar de forma autónoma y finalizando con la realización de un producto final. El proyecto descripto por las profesoras parece ser íntegramente planeado por el cuerpo docente: el producto final del proyecto es la realización de barras de cereal, y todo el proceso previo al producto final también es gestionado por las y los profesores.

En particular, con relación al producto final del proyecto experimentado, en el episodio 3 las profesoras se refieren a esta instancia, dando sustento a que las decisiones de planificación quedan sujetas a las disposiciones de los y las profesoras:

(...) de acá a fin de año que nos queda poco, un mes, vamos a hacer pero orientado a la alimentación por ejemplo de distintas enfermedades. Habíamos pensado los celíacos (...) la finalidad del proyecto era hablar todos de la enfermedad, cada una desde su punto de vista o de cada materia y después hacer bomboncitos para celíacos. (PB, comunicación personal, 1 de noviembre, 2019)

En relación con la elección de los temas que abordan los proyectos, en el episodio 14 las profesoras señalaron que también depende de la institución y grupos de las personas estudiantes. Hay escuelas que no forman parte del programa Escuela Promotora, pero solicitan al cuerpo docente trabajar con sus estudiantes algún proyecto en temáticas definidas por la institución. En cambio, en otras instituciones se permite acordar las temáticas de los proyectos con las personas estudiantes:

Por ahí acá lo podés llegar a hablar más con los chicos [en referencia a la institución en el que se desarrolla el proyecto de alimentación]. En la escuela ocho es como que viene más impuesto desde arriba [en referencia a las autoridades de la institución] (...) en la escuela catorce no (... ) Ahí eligen los temas que vamos a trabajar en proyecto los chicos. (PH, comunicación personal, 1 de noviembre, 2019)

En realidad nosotros elegimos este [en referencia al proyecto de alimentación] porque el año pasado ya lo había hecho y teníamos una base, porque arrancar de la nada iba a ser mucho más complicado (...) seguimos lo de la alimentación dijo la directora. (...) le conté un poco a las chicas [en referencia a los demás colegas] e incorporamos lo de Escuela Promotora y quedó así. El año que viene tal vez hagamos otra cosa. (PB, comunicación personal, 1 de noviembre, 2019)

En particular, con relación a los proyectos desarrollados en otras instituciones $\mathrm{PH}$, indicó lo siguiente en el episodio 12:

Igual más allá que estén o no acá te sirven un montón [en referencia al trabajo por proyecto]. Cada una de nosotras, en las otras escuelas, por ejemplo en la escuela ocho también se trabaja en proyectos (...) No es escuela promotora pero desde la dirección se trabaja con proyectos puntuales. Yo en el primer año que tengo trabajo con huerta y en segundo año por ejemplo 
trabajo la parte de arte, porque son proyectos que atraviesan y están fijos(...) Entonces si o si vos tenés que trabajar, el que vos quiera o arte o huerta (...) (PH, comunicación personal, 1 de noviembre, 2019)

En las intervenciones de las profesoras se destaca la necesidad de adquirir experiencia para el desarrollo de proyectos. El formar parte de una Escuela Promotora beneficia a profesores para tomar conocimiento de la gestión de una enseñanza por ABP para el estudio en escuelas donde no forman parte del programa. Las profesoras entrevistadas también contaban con experiencia sobre la gestión de una enseñanza por ABP antes de comenzar el programa Escuela Promotora, porque en la institución en la que desarrollan el proyecto de alimentación, propuso en años previos la implementación de este tipo de enseñanza. En el episodio 17, PB describió otros proyectos que desarrolló con las personas estudiantes:

Y el primer año que me propuso la directora hacer proyecto hice de huerta con los chicos de primero (...) conseguí cajones de frutas y verduras, tierra, plantines, las semillas. Nos vino a dar una charla los chicos del INTA y nos donó las semillas. (...) Era un proyecto solidario (...) Lo que hicimos después lo donamos al comedor [en referencia a un comedor comunitario de la ciudad] tiene aparte del comedor una huerta comunitaria. Entonces, todos los platines que nosotros logramos que crezcan se los llevaron. Y logramos que tres chicos hicieran huerta en su casa. (PH, comunicación personal, 1 de noviembre, 2019)

Si bien cada proyecto es irrepetible, tomar conocimiento sobre las experiencias de las profesoras y los profesores en cuanto a la gestión de los proyectos, resulta de relevancia para analizarlos y discutirlos. Estos saberes pueden contribuir a la formación de las personas docentes, siendo que esta formación es de manera monodisciplinar.

En particular, con relación a la gestión del proyecto sobre alimentación, en el episodio 8, las profesoras indicaron lo siguiente:

(...) comenzamos trabajando con las tablas nutricionales, después vimos que algunos decían gramos, y otros miligramos. Entonces empezamos viendo eso, qué era eso y fuimos a lo que era la historia de la medición. Arrancamos con la historia de la medición para después llegar a las diferentes medidas. Ahí si los hice trabajar en grupo con fotocopias. (...) cada unidad de medida los fui dividiendo en grupos y después lo pusimos en puesta en común. (PM, comunicación personal, 1 de noviembre, 2019)

Nosotros lo mismo, lo metimos (...) Yo arranco con los seres vivos, las funciones de los seres vivos, que está integrado por células (...) animal, vegetal y después se ven los sistemas de nutrición. Digestivo, respiratorio, circulatorio y ahí lo enganchamos, vimos alimentación. (PB, comunicación personal, 1 de noviembre, 2019)

Por eso es necesario adaptar. Porque a veces lo que tenés programado, lo tenés que adaptar a la situación. Yo en ciencias sociales arranco desde el inicio. Asique después que vimos los pueblos originarios en américa arranqué con la parte de alimentación porque es parte de su vida y de cómo se desarrollaron y fueron evolucionando. Entonces desde ahí vimos el origen de la papa, del tomate, de un montón de alimentos. (PH, comunicación personal, 1 de noviembre, 2019)

“(...) por ahí enganché por el lado de las unidades. Ahora estamos empezando a ver fracciones. También ahí, por el lado de las proporciones." (PM, comunicación personal, 1 de noviembre, 2019). "Hemos estado trabajando, un poco de ensayo y error porque somos todas nuevas. La profesora de Ciencias Naturales sería la única que tendría experiencia." (PH, comunicación personal, 1 nov. 2019)

Porque el año pasado la hice sola. Porque vos pensás ¿dónde lo metes? Pero sino haces un paréntesis con el contenido, y nosotros dividimos la carpeta, en el proyecto y otra los contenidos comunes (...) yo tengo dos veces por semanas, un día trabajamos en el proyecto, otro día avanzamos un poquito con los contenidos que tenemos que dar. (PB, comunicación personal, 1 de noviembre, 2019)

En este episodio se advierte cómo las profesoras gestionan la enseñanza por ABP. En primer lugar, destinan un periodo del estudio para desarrollar con el alumnado las nociones fundamentales que se requiere para el proyecto. Esto es por cada una de las materias involucradas, siendo que la distribución de horas destinadas para el estudio en cada espacio curricular se hace de manera fragmentada, es decir, no hay instancias en la que la totalidad de profesoras y profesores trabajen en conjunto con las personas estudiantes. También se destaca que las profesoras se preocupan por dar contenidos; la función de profesores parecería ser la de proporcionar los saberes y facilitar el material de estudio, mientras que el alumnado no parece tener suficiente espacio para 
indagar. Se percibe la preocupación de profesores por cumplir con el diseño curricular, pues la carpeta de las personas estudiantes es dividida en dos secciones: una de ellas se destina al trabajo por proyectos y la otra para el estudio de las nociones que se estipula en el diseño curricular. En esta institución, la enseñanza por ABP se realiza en un proceder híbrido entre una enseñanza tradicional de los saberes y el ABP.

En el episodio 20, PB manifestó su opinión acerca de la nueva propuesta de enseñanza y sus vínculos con el diseño curricular vigente:

Por más que vos metas algo en uno de los contenidos. Pero, ¿el resto? Los tenés que dar igual, los tienen que aprender igual. (...) No podés meter todo en el proyecto (...) Uno, dos, tres temas te pueden entrar ahí (...) ahora nosotras lo cortamos y seguimos con lo de salud porque ya se cortó. Entonces dentro del proyecto vos podés hacer dos o tres, involucrar así mismo un contenido del primero, un contenido del segundo trimestre y uno del tercero. En el año podés hacer el proyecto que tenga tres cosas. (...) (PB, comunicación personal, 1 de noviembre, 2019).

En la gestión de una enseñanza por ABP, las profesoras y los profesores parecen estar involucrados en dos problemáticas fundamentales, por un lado, buscar una temática que se ajuste al grupo de estudiantes $\mathrm{y}$, por otro, que esta temática se ajuste a los diseños curriculares de cada uno de los espacios curriculares implicados. Esto hace compleja la gestión de una enseñanza por ABP, siendo que en esta necesidad por satisfacer las dos problemáticas enunciadas se pierde la esencia de este tipo de enseñanza. El ABP no debe ser realizado como complemento a un determinado tópico, sino que tiene que ser el eje vertebrador del proceso de enseñanza -aprendizaje. Si bien, para las profesoras entrevistadas parece ser el proyecto el eje central del proceso de estudio, sigue en ellas la preocupación de cómo articular en el proyecto los contenidos estipulados en el diseño curricular para cada disciplina. Resulta complejo establecer proyectos integrados cuando los contenidos siguen siendo listados y fragmentados en disciplinas sin relación entre ellos y en general sobrecargados, repetitivos o desfasados (Pozuelos y Rodríguez, 2008). Según Coll (2006), se requiere generar proyectos que eviten el enciclopedismo dominante en las propuestas curriculares actuales, siendo que obstaculizan la enseñanza de calidad, fomentando la formación memorística y superficial con bajo impacto, más allá de las aulas y situaciones en las que se aprendió.

\subsection{El lugar dela profesorado en una enseñanza por proyectos}

Iniciar un proceso educativo innovador conlleva a modificar las tareas y responsabilidades habituales del profesorado. En referencia a esto, en el episodio 17, PB indicó lo siguiente:

Es un trabajo más para nosotras en realidad (...) En estas dos horas que nos reunimos, y está buenísimo porque aparte hablamos de otros temas (...) No solo lo usamos para proyecto sinceramente, sino que tratamos de ver individualmente cómo va cada uno [en referencia a los estudiantes]. (PB, comunicación personal, 1 de noviembre, 2019)

PB destaca que la enseñanza por ABP demanda mayor trabajo al profesorado que las prácticas habituales. Brush y Saye (2008) señalan que la enseñanza por ABP es un reto para profesores, dado que requieren de espacios para planificar y difundir el ABP de manera eficiente. En este sentido, las personas docentes tienen un rol dual, por un lado, deben desempeñarse como orientadoras del aprendizaje y de los procesos del estudiantado y, por otro, deben dejar que ellos y ellas adquieran autonomía y responsabilidad en su aprendizaje (Johari y Bradshaw, 2008). A su vez, en el episodio 17, PB destaca la importancia de contar con espacios en los que se puedan reunir profesores, para conversar del proyecto, y de la trayectoria individual de cada persona estudiante.

En relación con la gestión del proyecto que se encontraban desarrollando las profesoras, estas destacaron lo siguiente en el episodio 15:

El profe de educación física por ejemplo, hablándoles desde la salud del cuerpo qué cosas están buenas, qué alimentos pueden, según los deportes que hagan como para la vida diaria. Qué cosas están buenas comer, qué no. (...) Vos trabajaste el tema 
del óvalo nutricional [en referencia a PB] y todo eso. Y eso también lo retoma el profe de educación física (...) En artística estuvieron trabajando con las partes del cuerpo (...) (PH, comunicación personal, 1 de noviembre, 2019)

Y lo de construcción era de (...) Bulimia, anorexia (...) Ellos no vienen [en referencia a los profesores que no pueden asistir al encuentro] entonces todo vía whatsApp (...) Y al principio le decíamos, tienen que hacer las rúbricas [en referencia a los profesores que participan en el proyecto y no pueden asistir a las reuniones] y algunos estaban re-perdidos porque no sabían qué tenían que hacer. Al menos por lo menos una vez por mes tendrían que pagarle para poder reunirse. Si es de todas las materias tienen que estar todas. (PB, comunicación personal, 1 de noviembre, 2019)

En este último fragmento de protocolo se destaca la complejidad a la que se enfrenta el profesorado en cuanto a coordinar para el desarrollo de los proyectos. Profesores son convocados a participar de los encuentros si tienen un tiempo de dedicación en el curso de cuatro horas, si no, deben integrar los proyectos, pero no reciben remuneración para poder asistir a los encuentros. Esto constituye una restricción al momento de la gestión del proyecto, siendo que la comunicación se establece mediante medios no presenciales, lo que dificulta la coordinación y planificación de una enseñanza por ABP.

En particular, con relación al proyecto que desarrollaron en conjunto las profesoras, en el episodio 4, PB destacó su experiencia en desarrollar un proyecto de manera individual y de manera conjunta con otras y otros profesores:

El año pasado la directora me propuso hacer uno porque le había llegado la invitación a ella y lo hice yo sola, sin escuela promotora porque todavía no estaba, y también fue lo de las barritas. Pero yo solamente desde el punto de vista de Ciencias Naturales. Bueno, les dí todos los contenidos, solo de ciencias naturales. No pude incluir matemática. Les dí los tipo de alimentos, lo que contenían, dónde los podían encontrar. Lo mismo que hice este año, pero sola. (PB, comunicación personal, 1 de noviembre, 2019)

En este último episodio se advierte la importancia que PB le asigna al trabajo cooperativo entre el profesorado. La profesora destaca que el trabajo monodisciplinar solo permite atender de forma parcializada la temática de los proyectos.

\subsection{El lugar del estudiantado en una enseñanza por proyectos}

En una enseñanza por ABP el rol del profesorado y del estudiantado dista bastante del habitual. En particular, en el ABP las personas estudiantes ocupan el lugar de solucionar problemas, generar preguntas, diseñar planes, recopilar datos, establecer conclusiones, exponer sus resultados y crear un producto final (Blumenfeld et al., 1991). Durante la elaboración del proyecto es deseable que el estudiantado manifieste sus dudas, formule hipótesis de trabajo, confronte alternativas, busque información y llegue a acuerdos finales que posibiliten la conclusión del trabajo. En una enseñanza por ABP, el profesorado deja de constituir poseedor del saber, para convertirse en guía y acompañante de las personas estudiantes (Ayerbe y Perales, 2020). En el episodio 7, las y los profesores destacan la importancia de que las y los estudiantes experimenten ABP, porque permitiría dar sentido a los saberes que se proponen estudiar en el diseño curricular para cada materia en interacción con otras disciplinas. También el profesorado destaca su percepción acerca de la actividad de las personas estudiantes: “(...) Primero el aprendizaje de los chicos es diferente. Lo que tiene, lo que ven en una materia, en otra y otra lo mismo. Estamos todos enfocados en lo mismo." (PB, comunicación personal, 1 de noviembre, 2019). "Al principio te decían, ¡no! ¿Vamos a trabajar de vuelta, otra vez con esto?” (PM, comunicación personal, 1 de noviembre, 2019)

Ya lo vimos en naturales, ¿̇otra vez en matemática? Que aprendan a que no solo sirve la escuela para eso y nada más (...) que se puede ver de diferentes lugares (...) Vos le das un contenido y te dicen: ¿ para qué te sirve? Vos ese contenido fíjate que lo podés ver desde diferentes puntos de vista. ¿Para qué matemática? Bueno, matemática te sirve para poder alimentarte bien también. No es solo hacer una cuenta. (PB, comunicación personal, 1 de noviembre, 2019)

Calcular las calorías, las proporciones, saber lo que están comiendo, el aprender a leer una etiqueta. El saber cómo se conserva el alimento. ¿Cuáles necesitan heladera? ¿Cuáles no? Y si voy a comer un alimento ver la fecha de vencimiento. 
Cuando se pusieron a trabajar hubo lavado de manos y después hubo alcohol en gel (...) Las cosas mínimas, para que ellos puedan ir incorporándolas a sus vidas. (PH, comunicación personal, 1 de noviembre, 2019)

En este último protocolo, PM destaca que el estudiantado manifiesta su disconformidad cuando la totalidad de las y los profesores, en sus clases, refieren a la misma temática. A su vez, PB resalta la importancia del estudio por $\mathrm{ABP}$, siendo que permite a las personas estudiantes, encontrar sentido a los saberes que estudian en la escuela, comprendiendo su funcionalidad en el hacer del proyecto.

En referencia al vínculo del estudiantado con el ABP, PB indicó lo siguiente:

(...) Está bueno porque los sacás de dar los contenidos los enganchas desde ese lado. Y ver que ese contenido que nosotros vimos (...) se puede trabajar de otra manera (...) en una lámina por ejemplo, el que sabe dibujar dibuja, el que sabe escribir escribe, uno tiene linda letra, el otro pinta. (PB, comunicación personal, 1 de noviembre, 2019)

PB destaca la importancia de que las personas estudiantes vivan experiencias en $A B P$, siendo que potenciaría sus habilidades. Esto se encuentra en correspondencia con lo indicado por Mergendoller, Maxwell y Bellisimo (2006), quienes indican que el ABP resulta positivo en la adquisición de conocimientos, el desarrollo de habilidades tales como la colaboración, la resolución de problemas y el pensamiento crítico.

\subsection{La evaluación en una enseñanza por proyectos}

La evaluación en la enseñanza por ABP requiere de modificaciones con relación a la enseñanza tradicional. Rekalde y García (2015) destacan que la evaluación en una enseñanza por ABP:

se debe abordar desde su sentido más holístico, poniendo en juego técnicas, instrumentos y dinámicas de evaluación que sintonicen los resultados del aprendizaje con las evidencias que el alumnado aporta, dando cabida, no sólo, a la heteroevaluación, sino a instrumentos de autoevaluación y evaluación entre pares (p. 230).

En este sentido, los autores destacan que la evaluación debe generar dinámicas que permitan la introspección no solo de estudiantes, sino también de profesores, las familias y el entorno. En el episodio 2, las profesoras se refirieron a la evaluación en una enseñanza por ABP del siguiente modo: “(...) El tema de rúbricas empezamos a aplicarlo, para poder evaluarlos. (...) Nosotros en un principio teníamos 11 ítems con el que teníamos que evaluar (...)” (PH, comunicación personal, 1 de noviembre, 2019). "Eran 11 ítems ahora hay 4." (PB, comunicación personal, 1 de noviembre, 2019). "Porque en este caso nosotras trabajamos el tema de alimentación saludable con todas las materias. Desde inglés, educación física, artística construcción, naturales, matemáticas, sociales, y práctica del lenguaje. Todas trabajamos, entonces había que aunar criterios." (PH, comunicación personal, 1 de noviembre, 2019). "Que sea para el conjunto de todas las materias. Ninguno podía ser específico.” (PB, comunicación personal, 1 de noviembre, 2019).

La idea de evaluación que se propone en una enseñanza por ABP parece estar centrada únicamente en la actividad de las personas estudiantes. En el episodio 2 se destaca la problemática de las profesoras en acordar criterios para poder evaluar al estudiantado. Las profesoras indican que los documentos ministeriales establecen 11 ítems para evaluar a las y los estudiantes en una enseñanza por ABP, pero para poder desarrollar las evaluaciones, el profesorado acordó criterios estableciendo 4 ítems. En este sentido, en el episodio 9 las profesoras volvieron a referirse a la temática de evaluación en una enseñanza por ABP:

Lo mismo para evaluar. Porque vos el resto de la materia la vas evaluando con un número pero en el proyecto no. Entonces para todos fue un cambio importante. Por eso el tema de poder coincidir en tres conceptos que podamos evaluar todas, porque sino se complica muchísimo. (PH, comunicación personal, 1 de noviembre, 2019)

"Igualmente nosotras le pusimos número." (PB, comunicación personal, 1 de noviembre, 2019)

Como para que ellos tengan una idea de lo que equivale (...) Se les da trabajos extras [en referencia a los estudiantes que no aprueban], como para que pueda (...) finalmente se llega a una nota ponderativa, si a lo largo del año y vos ves que estaba 
para un seis, pero al final remontó y vos ves que va creciendo, la nota final va a ser un siete. (PH, comunicación personal, 1 de noviembre, 2019)

"Por eso estos parámetros (...) que sea algo medio general. (...) Chico por chico tenés que hacer eso. Cada docente entregaba la rúbrica de su materia (...) después ella [En referencia a

] las tiene que juntar en esto.” (PB, comunicación personal, 1 de noviembre, 2019)

En este último episodio, se destaca la evaluación individualizada que debe realizar el profesorado de cada estudiante, aunando criterios con la totalidad de los y las profesoras que participan del proyecto. Además, se destaca la actividad del PAT en reunir la evaluación de la totalidad de profesores por cada estudiante.

En el sistema actual de la escuela secundaria convive la evaluación de proyectos por rúbricas y el formato tradicional de evaluación de la escuela secundaria, en el que se asigna una calificación numérica (en la escala de 1 a 10) a cada estudiante por cada materia. Esto hace que las calificaciones se registren en un boletín con las notas tradicionales. PB destaca la necesidad de indicar una calificación numérica para que las personas estudiantes comprendan sus calificaciones, siendo que ellas también se deben habituar a otro sistema de evaluación.

\subsection{La formación del profesorado en ejercicio sobre proyectos}

Uno de los aspectos que profundizó la entrevistadora es la formación que recibió el profesorado para gestionar una enseñanza por ABP. En el primer episodio, las profesoras destacaron su formación monodisciplinar y las dificultades para poder desarrollar una enseñanza interdisciplinaria como se solicita desde las nuevas disposiciones ministeriales. Al respecto, en el episodio 1, PH indicó:

(...) creo que como todos nos formamos es en una burbuja ideal, cuando salimos [en referencia cuando el profesor se recibe] Y las estrategias que nos enseñaron (...) la mayoría en tiempo no las podemos aplicar. Nosotras es el primer año que trabajamos en proyecto todas conjuntas. Hemos ido a perfeccionamiento y estamos ensayo y error. ( $\mathrm{PH}$, comunicación personal, 1 de noviembre, 2019)

En el episodio 16, las profesoras hicieron referencia a los cursos que realizaron para formarse en ABP. PH profundizó en un curso que realizó y el que se encontraba gestionado por tres profesores de las disciplinas: Biología, Educación Física y Geografía. En relación con el curso, PH indicó lo siguiente:

(...) fue un curso de tres clases (...) Ahí saqué para socializar la información que nos dio porque por ejemplo cómo hacer el paso a paso de un $\mathrm{ABP}($...) Yo lo trabajé con los profesores que pertenecen a la escuela. (...) ella no pudo ir, no la autorizaron [en referencia a PB] (...) Tuvimos que armar un esquema, el eje del proyecto. Cómo sería la problemática, de dónde arrancás y cómo lo llevaría a cabo dentro del aula. En el caso nuestro podría abarcar todos los años. De primero a quinto, a sexto. Cada uno de los años trabajar algo diferente. (PH, comunicación personal, 1 de noviembre, 2019)

En este episodio $\mathrm{PH}$ destacó la imposibilidad de PB en desarrollar el curso para capacitarse, dado que fueron convocados algunos y algunas profesoras de la institución. En este protocolo se manifiesta la disparidad en la formación del profesorado en ABP, quienes tienen una formación de base monodisciplinar.

\section{CONCLUSIONES}

En esta investigación se exponen las condiciones y restricciones que manifiestan experimentar profesoras de una escuela secundaria argentina al promover un ABP. Las profesoras entrevistadas procuran desarrollar proyectos que no solo impliquen comprender el funcionamiento de diversos saberes en el desarrollo de este, sino también, que los saberes trasciendan a las familias del estudiantado. En particular, considerando que las personas estudiantes de la institución, en la que desarrollan funciones docentes las profesoras entrevistadas, pertenecen a sectores vulnerables de la sociedad. 
Los resultados del análisis reportado en este trabajo indican que las personas estudiantes y profesoras en la enseñanza por ABP, conservan los lugares que la enseñanza tradicional les ha asignado. El profesorado tiene un rol preponderante en el desarrollo de proyectos: son quienes deciden qué proyectos desarrollar, qué saberes se requieren estudiar y desde qué materiales hacerlo. Esto reduce notablemente el lugar del estudiantado en el desarrollo de proyectos. En particular, en una enseñanza por ABP se pretende que las pesonas estudiantes reinterpreten los saberes convenientemente para su empleo en distintas situaciones o nuevas necesidades y problemas, trascendiendo la simple comprensión o proceder mecánico según fueron enseñados.

Otro de los resultados que se destaca del análisis de la entrevista, es que la gestión de una enseñanza por $\mathrm{ABP}$ es un reto que despierta compromiso en sus participantes, pero también incrementa los esfuerzos, en particular del profesorado, para sostener el estudio. La enseñanza por ABP implica planificación, tiempo para el trabajo en equipo, intercambio de información entre colegas, etc., en una estructura de escuela que no se encuentra aún preparada para propiciar un eficaz desarrollo de este tipo de enseñanza. Los documentos ministeriales propugnan una enseñanza por $\mathrm{ABP}$, pero la estructura de la escuela secundaria argentina continúa conservando las mismas características: disciplinas segmentadas en las que se establecen los saberes a estudiar en determinados espacios temporales.

Con respecto a la formación del profesorado para la gestión de $\mathrm{ABP}$, en la entrevista se destaca que las personas docentes no tienen acceso de manera homogénea a la formación. Los cursos de capacitación son habilitados para docentes que reúnen determinados requisitos. Acceder a estos cursos es fundamental para el profesorado de la escuela secundaria, puesto que la especialización en que son formados, basada en la parcialización del saber, atenta contra un estudio interdisciplinario, tal como requiere emprender cualquier proyecto del mundo real.

\section{Recomendaciones}

Establecer los aspectos propicios para una enseñanza por ABP requiere establecer los elementos que se presentan como obstáculos a las iniciativas de cambio y planear las condiciones que deberían considerarse ante la complejidad que implica la transformación que se pretende. Una de las condiciones que debe ser considerada, es la formación del profesorado. Sin embargo, resulta complejo plantear cambios en los sistemas de formación de profesores si el currículum y las formas de trabajo en la escuela secundaria no experimentan cambios sustantivos, considerando también que estos cambios son poco posibles sin personas docentes capacitados para producirlos. La escuela secundaria argentina, en su desarrollo histórico, se estructuró en torno a tres disposiciones básicas: la clasificación de los currículos, el principio de designación de profesores por especialidad y la organización del trabajo de docentes por horas de clase (Terigi, 2008). Esta estructura de la escuela secundaria atenta contra el buen desarrollo de una enseñanza por ABP.

El ABP implica cambios en la manera de aprender de las personas estudiantes, la manera de enseñar del profesorado y el papel de las familias frente a la educación regulada. Es necesario continuar investigando y generar espacio para la formación del profesorado, dado que uno de los aspectos esenciales a atender en una enseñanza por $\mathrm{ABP}$, es cambiar el lugar que la enseñanza tradicional le ha asignado a las personas estudiantes y profesoras; y que parece estar arraigada en las profesoras entrevistadas, pues son quienes manifiestan planificar los proyectos, diseñar actividades y facilitar material al estudiantado. Es preciso idear propuestas compatibles con una enseñanza por ABP para la formación de las y los profesores, que conduzcan a cambiar la propia mirada sobre su profesión y las personas estudiantes. En particular, se requiere una enseñanza centrada en el tratamiento de problemas en el que interactúen diversas disciplinas, se seleccione y se sintetice información, se confeccionen productos con argumentaciones sólidas, etc. Esto conduce a una formación que no se puede fundamentar en la reproducción o almacenamiento de información, sino que busca su funcionalidad y reconstrucción en diversas situaciones. 


\section{REFERENCIAS}

Ayerbe, J. y Perales, F. (2020). «Reinventa tu ciudad»: aprendizaje basado en proyectos para la mejora de la conciencia ambiental en estudiantes de Secundaria. Enseñanza de las Ciencias, 38(2), 181-203. doi: https://doi.org/10.55 $65 /$ rev/ensciencias. 2812

Bell, T., Urhahne, D., Schanze, S. y Ploetzner, R. (2010). Collaborative inquiry learning: Models, tools, and challenges. International Journal of Science Education, 3(1) 349-377. doi: https://doi.org/10.1080/09500690802582241

Blumenfeld, P., Soloway, E., Marx, R., Krajcik, J., Guzdial, M. y Palincsar, A. (1991). Motivating projectbased learning: Sustaining the doing, supporting the learning. Educational Psychologist, 26(3-4), 369-398. doi: https://doi.org /10.1080/00461520.1991.9653139

Brush, T. y Saye, J. (2008). The Effects of Multimedia-Supported Problem-based Inquiry on Student Engagement, Empathy, and Assumptions About History. Interdisciplinary Journal of Problem-Based Learning, 2(1), 1- 56. doi: https://doi.org/10.7771/1541-5015.1052

Coll, C. (2006). Lo básico en la educación básica. Reflexiones en torno a la revisión y actualización del currículo escolar. Revista Electrónica de Investigación Educativa, 8(1), 1-17. Recuperado de: http://redie.uabc.mx/vol8no1/con tenido-coll.html

Dewey, J. (1997). How we think. New York, EE. UU.: Dover Publications.

Dirección de Educación Secundaria de la Provincia de Buenos Aires. (2017). Saberes coordinados y aprendizaje basado en proyectos: hacia una enseñanza compartida para lograr aprendizajes integrados. Documentos de actualización curricular. Buenos Aires: Gobierno de la provincia de Buenos Aires. Recuperado de: https://bit.ly/30KNfzH

Eceizabarrena, R. (2018). El aprendizaje a través de proyectos suprasignaturas en el contexto real. En A. Eizaguirre, M. Bezanilla y A. García-Olalla (Coords.). Innovación docente en Educación Superior (pp. 79-92). Madrid, España: Pearson.

Ginsburg, H. y Opper, S. (1987). Piaget's theory of intellectual development. New York, EE. UU.: Pearson.

Gobierno de la Provincia de Buenos Aires (2018). Experiencia pedagógica para la Escuela Secundaria de la Provincia de Buenos Aires Resolución 5222/18. Dirección General de Cultura y Educación. Recuperado de http://abc.gov. ar/nuevoformatosecundaria/normativa

Hernández, R., Fernández, C. y Baptista, P. (2014). Metodología de la investigación. México D.F, México: Editorial Mc Graw Hill.

Johari, A. y Bradshaw, A (2008). Project-based learning in an internship program: A qualitative study of related roles and their motivational attributes. Educational Technology Research and Development, 56, 329-359. doi: https:/ /doi.org/10.1007/s11423-006-9009-2

Jones, N., Rassmussen, C. y Moftt, M. (1997). Real-life problem solving: A collaborative approach to interdisciplinary learning. Washington, EE. UU.: American Psychological Association.

Kilpatrick, W. (1918). The project method: the use of the purposeful act in the educative process. Nueva York, EE. UU.: Teachers College, Columbia University.

Kilpatrick, W. (1967a) La filosofía de la educación desde el punto de vista experimentalista. En W. Kilpatrick, F. Breed, H. Horne y M. Adler (Eds.), Filosofía de la Educación (pp. 15-74). Buenos Aires, Argentina: Editorial Losada.

Kilpatrick, W. (1967b) La teoría pedagógica en que se basa el programa escolar. En W. Kilpatrick, H. Rugg, G. Washburne y F. Bonner (Eds.), El nuevo programa escolar (pp. 39-72). Buenos Aires, Argentina: Editorial Losada.

Kilpatrick, W., Rugg, H., Washburne, C. y Bonner, F. (1967). El nuevo programa escolar. Buenos Aires, Argentina: Editorial Losada.

Lacueva, A. (1998). La enseñanza por proyectos: ¿mito o reto? Revista Iberoamericana de Educación, 16, 165-187. Recuperado de https://rieoei.org/historico/oeivirt/rie16a09.pdf

Larmer, J. y Mergendoller, J. (2010). Seven Essentials for Project-Based Learning. Educational Leadership, 68(1), 34-37. Recuperado de https://bit.ly/2SAivgf 
López, A., Ugalde, A., Rodríguez, P. y Rico, A. (2015). La enseñanza por proyectos: una metodología necesaria para los futuros docentes. OPCION, 31(1), 395 - 4130 Recuperado de https://bit.ly/33AY49g

Majó, F. y Baqueró, M. (2014). 8 ideas Clave. Los proyectos interdisciplinarios. Barcelona, España: GRAÓ.

Mejía, J. (2011). Problemas centrales del análisis de datos cualitativos. Revista Latinoamericana de Metodología de la Investigación Social, 1, 47-60. Recuperado de: http://www.relmis.com.ar/ojs/index.php/relmis/issue/view/2/s howToc

Mergendoller, J., Maxwell, N. y Bellisimo, Y. (2006). The effectiveness of problem-based instruction: A comparative study of instructional methods and student characteristics. Interdisciplinary Journal of Problem-based Learning, 1(2), 49-69. doi: https://doi.org/10.7771/1541-5015.1026

Ministerio de la Nación (2006). Ley de Educación Nacional N²6.206. Boletín Oficial.

Ministerio de Educación de la Nación (2017). Marco de Organización de los Aprendizajespara la Educación Obligatoria Argentina (MOA). Anexo Resolución CFE Nro 330/17. Recuperado de: http://www.bnm.me.gov.ar/giga1/nor mas/RCFE_330-17.pdf

Pozuelos, F. y Rodríguez, F. (2008). Trabajando por proyectos en el aula. Aportaciones de una investigación colaborativa. Investigación en la Escuela, 66, 3-33. doi: https://doi.org/10.12795/IE.2008.i66.01

Rekalde, I. y García, J. (2015). El Aprendizaje Basado en Proyectos: un constante desafío. Innovación Educativa, 25, 219-234. doi: https://doi.org/10.15304/ie.25.2304

Savin, M. y Major, C. H. (2013). Qualitative research: The essential guide to theory and practice. Nueva York: Routledge.

Terigi, F. (2007). Exploración de una idea. En torno a los saberes sobre lo escolar. En R. Baquero, G. Diker y G. Frigerio. (Comps.), Las formas de lo escolar (pp. 99-118). Buenos Aires, Argentina: Del estante.

Terigi, F. (2008). Los cambios en el formato de la escuela secundaria argentina: por qué son necesarios, por qué son tan difíciles. Propuesta Educativa, 29(1), 63-71. Recuperado de: https://bit.ly/3lhd0iK

Thomas, J. (2000). A review of research on Project-based learning. California, EE. UU.: Autodesk Foundation.

Torrego, L. y Méndez, R. A. (2018). Un acercamiento al aprendizaje basado en proyectos, cien años después de «Te Project Method», de WH Kilpatrick. Revista Electrónica Interuniversitaria de Formación del Profesorado, 21(2), $1-12$.

Valles, M. (1999). Técnicas cualitativas de investigación social. Madrid, España: Sintesis Editorial.

Vergara, J. (2016). Aprendo porque quiero. El Aprendizaje Basado en Proyectos (ABP), paso a paso. Madrid, España: Biblioteca Innovación Educativa, Ediciones SM.

Vygotsky, L. (1962). Thought and language. Cambridge, Reino Unido: MIT Press. 